\title{
El rol de la memoria de trabajo y la atención sostenida en la generación de inferencias explicativas
}

\author{
The role of working memory and sustained attention in \\ explanatory inference generation
}

\author{
Juan Pablo Barreyro a, ${ }^{\mathrm{*}}$, Irene Injoque-Ricle ${ }^{\mathrm{a}}$, Jésica Formoso ${ }^{\mathrm{a}}$, Debora I. Burin ${ }^{\mathrm{a}}$ \\ a Departamento de Procesos Básicos, Instituto de Investigaciones, Facultad de Psicología, \\ Universidad de Buenos Aires; CONICET, Argentina
}

\section{Resumen}

El propósito de este trabajo consistió en estudiar la relación entre la memoria de trabajo, la capacidad de sostener la atención y la generación de inferencias explicativas-repositivas en la comprensión de textos expositivos. Para ello, 120 alumnos universitarios leyeron dos textos expositivos del área de las ciencias naturales y completaron un cuestionario de generación de inferencia, junto con cuatro pruebas de memoria de trabajo verbal y dos pruebas que miden la capacidad de sostener la atención sobre una tarea. Los resultados indicaron que el componenente ejecutivo de la memoria de trabajo verbal tienen un efecto directo sobre la generación de inferencias, mientras la capacidad de sostener la atención y el almacenamiento de información en la memoria de trabajo verbal tienen un efecto indirecto mediado por el componente ejecutivo. Esto sugiere que las diferencias individuales en la generación de inferencias están vinculadas a las diferencias inviduales en la memoria de trabajo, y también a las diferencias individuales en la capacidad de sostener la atención.

Palabras clave: comprensión, textos expositivos, inferencias, memoria de trabajo, atención sostenida.

\section{Para citar este artículo:}

Barreyro, J., Injoque-Ricle, I., Formoso, J., \& Burin, D. (2017).El rol de la memoria de trabajo y la atención sostenida en la generación de inferencias explicativas. Liberabit, 23(2), 233-245. doi: 10.24265/liberabit.2017.v23n2.05

\begin{abstract}
The aim of this work was to study the relationship between working memory, sustained attention and explanatory inference generation in expository texts comprehension. To this end, 120 undergraduate students read two expository texts from natural sciences and completed an inference generation questionnaire, along with four verbal working memory tasks and two sustained attention tasks. The results showed that the executive component of verbal working memory has a direct effect on inference generation, while sustained attention and information storage in verbal working memory have an indirect effect mediated by the executive component. This supports the idea that individual differences in inference generation are related to individual differences in working memory, and also to individual differences in the ability to sustain attention.
\end{abstract}

Keywords: comprehension, expository texts, inferences, working memory, sustained attention.

Este es un artículo Open Access bajo la licencia Creative Commons Atribución-NoComercial-CompartirIgual 4.0

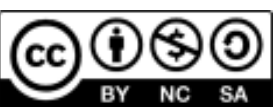


Desde un punto de vista cognitivo, la comprensión del texto se define como una de las más complejas actividades cognitivas humanas y supone la construcción de una representación coherente en una memoria episódica del significado global del texto (Cornoldi \& Oakhill, 2013; Kintsch \& van Dijk, 1978; Van Dijk \& Kintsch, 1983). Para lograr esta representación, el lector debe conectar ideas provenientes de diferentes partes del texto e integrarlas (Abusamra, \& Joanette, 2012), esto es, debe realizar inferencias y conexiones entre la información literal explícita (Elbro \& Buch-Iversen, 2013; van den Broek \& Kendeou, 2008). La generación de inferencias se refiere a la activación de información que no se encuentra explícitamente enunciada durante la lectura para dar mayor coherencia a la oración focal que se está leyendo (Carlson et al., 2014; Freed \& Cain, 2016; van den Broek, Risden, Fletcher, \& Thurlow, 1996).

En educación, los textos expositivos son de gran importancia, ya que constituyen una herramienta central para el aprendizaje (Vidal-Abarca, Martínez, \& Gilabert, 2000). El propósito de estos consiste en informar al lector acerca de nuevos conceptos, realidades abstractas y aportar información técnica necesaria para una tarea, lo que convierte a su comprensión en un desafío (Singer \& O'Connell, 2003; Snow, 2002).

Un tipo particular de inferencias relevantes en la comprensión de textos son las inferencias causales (Barth, Barnes, Francis, York, \& Vaughn, 2015; Graesser, Singer, \& Trabasso, 1994; Kendeou, Smith, \& O’Brien, 2013), que si bien surgieron en el estudio de la comprensión de narraciones (van den Broek, Rohleder, \& Narváez, 1996) son importantes para la comprensión de textos expositivos (Saux, Irrazabal, \& Burin, 2014) ya que activan información previamente presentada por el texto o desde el conocimiento previo para integrar la información proveniente de distintas oraciones con el objetivo de alcanzar la coherencia global del texto (Graesser \& Bertus, 1998; Leon \& Peñalba, 2002; Singer,
Harkness, \& Stewart, 1997; van den Broek, Virtue, Everson, Tzeng, \& Sung, 2002). En la comprensión de textos expositivos se proponen tres tipos principales de inferencias causales (van den Broek et al., 2002): las inferencias hacia atrás o explicativas (que pueden ser elaborativas o de reposición de información), las inferencias asociativas y las inferencias hacia delante (o predictivas). Las inferencias explicativas son aquellas que proporcionan las razones que explican por qué ocurre algo dentro de la trama textual, y cuando se generan el lector produce vínculos entre la oración que está leyendo en ese momento y los factores causales antecedentes. Las inferencias explicativasreposicionales sirven para unir información de la oración que se está leyendo con la información previamente presentada en el texto, mientras que las inferencias explicativas-elaborativas establecen conexiones entre la información que está siendo leída y el conocimiento previo del lector.

La memoria de trabajo es un sistema de procesamiento y almacenamiento concurrente de información on-line al servicio de tareas cognitivas complejas, como la comprensión (Baddeley, 2010; Unsworth \& McMillan, 2013) Según el modelo clásico de Baddely y Hitch (1974), está formada por tres subsistemas: el ejecutivo central, el bucle fonológico y la agenda viso-espacial. El ejecutivo central es un sistema de atención y control que fiscaliza y regula la información, asigna los recursos a los sistemas y a las tareas (Baddeley, 2010). El bucle fonológico almacena por breves períodos de tiempo una cantidad limitada de información verbal, y la agenda viso-espacial realiza la misma acción con información visual y espacial (Baddeley, 2010). En la comprensión del texto y en la generación de inferencias, la memoria de trabajo juego un rol crucial (Bohn-Gettler \& Kendeou, 2014; Prat, Seo, \& Yamasaki, 2016), ya que permite que se almacenen las representaciones de los procesos mientras el lector integra o activa información sucesiva del texto. 
La atención sostenida se refiere a la capacidad de mantener durante un periodo prolongado de tiempo el foco de atención sobre una determinada tarea (Rebollo \& Montiel, 2006). Esta capacidad permite los procesos de repaso, tanto verbales como viso-espaciales, que mantienen activas las representaciones almacenadas en los componentes pasivos de la memoria de trabajo, evitando el decaimiento espontáneo de la huella (Awh \& Jonides, 2001). Contar con capacidad disponible de sostenimiento de la atención permite focalizar la misma para poner en marcha procesos necesarios de almacenamiento y recuperación información de la memoria -tanto de trabajo como de largo plazo-, y resolver problemas (Diamond \& Goldman-Rakic, 1989). La capacidad de sostener la atención también cumple un rol importante en la comprensión. Estudios en pacientes con trastorno por déficit de atención con hiperactividad encontraron que éstos presentan grandes dificultades en la comprensión de textos (Brock \& Knapp, 1996; Hecker, Burns, Katz, Elkind, \& Elkind, 2002).

Las investigaciones sobre diferencias individuales han centrado sus objetivos en vincular a la memoria de trabajo con la comprensión de textos (Barreyro, Cevasco, Burin, \& Molinari, 2012; Bohn-Gettler \& Kendeou, 2014; Currie \& Cain, 2015; Prat et al., 2016; Van Dyke, Johns, \& Kukona, 2014; Wang \& Gathercole, 2013), pero pocas se han centrado en los textos expositivos (Budd, Whitney, \& Turley, 1995; Graesser, Leon, \& Otero, 2002; van den Broek et al., 2002). En relación a las capacidades atencionales muy pocas investigaciones se han centrado en estudiar su vínculo con la comprensión, y las que se encuentran se han centrado en ver la incidencia de patologías atencionales (McVay \& Kane, 2012).

El propósito de este trabajo consiste en estudiar el rol de la memoria de trabajo, específicamente los subsistemas vinculados a la comprensión como ser el bucle fonológico y el ejecutivo central, y la capacidad de sostener la atención en la generación de inferencias explicativas-repositivas en la comprensión de textos expositivos.

\section{Método}

\section{Muestra}

Participaron 120 alumnos del primer y segundo año de la Facultad de Psicología de la universidad de Buenos Aires, de forma voluntaria y anónima (26 varones -21.67\%-, 94 mujeres), con un promedio de edad de 21.46 años $(D E=3.07)$, de entre 18 y 30 años. Todos ellos, nativos del español, que dieron su consentimiento firmado para participar en la investigación.

\section{Materiales}

\section{Tareas de memoria de trabajo}

Se administraron tareas de almacenamiento de información verbal en la memoria de trabajo de la Batería Informatizada de Memoria de Trabajo Verbal (BIMeTV, Barreyro, Injoque Ricle, Formoso, \& Burin, s.f.): 1) Amplitud de Dígitos Directos, que consiste en presentar visualmente en una pantalla de computadora, de a uno por vez, números del dos al nueve. El sujeto tiene que retener dichos números y cuando aparezca la palabra «recuerdo» tiene que indicar en una matriz de números cuáles le fueron presentados, en el exacto orden en el que aparecieron. 2) Amplitud de Letras, que tiene el mismo procedimiento que amplitud de dígitos, salvo que, en lugar de números, los estímulos son letras. Se administraron también tareas que tienen como objetivo evaluar la capacidad de almacenamiento y procesamiento concurrente de información verbal en la memoria de trabajo. 3) Amplitud Rápida, que consiste en presentar al sujeto una serie de letras no se le indican cuántas- y su objetivo es en recordar, respetando el orden, las últimas letras de la serie -en el caso del nivel 2, las últimas dos letras-, cuando aparece la palabra «recuerdo». 4) Ordenamiento Letra-Dígito, que consiste en presentar en la pantalla de la computadora números y letras mezclados, $\mathrm{y}$ 
cuando aparece la palabra «recuerdo» el sujeto debe indicar en una matriz de números y letras, primero las letras en orden alfabético, y luego los números, que le fueron presentando, en orden creciente. En la Figura 1 se puede observar un ejemplo de Nivel 3 de todas las pruebas de memoria.
Amplitud de dígitos directos

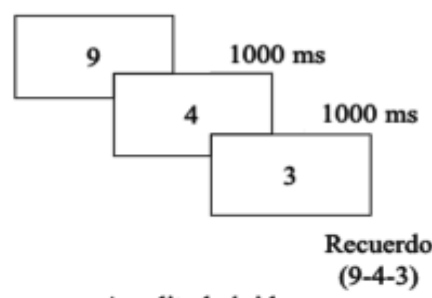

Amplitud rápida

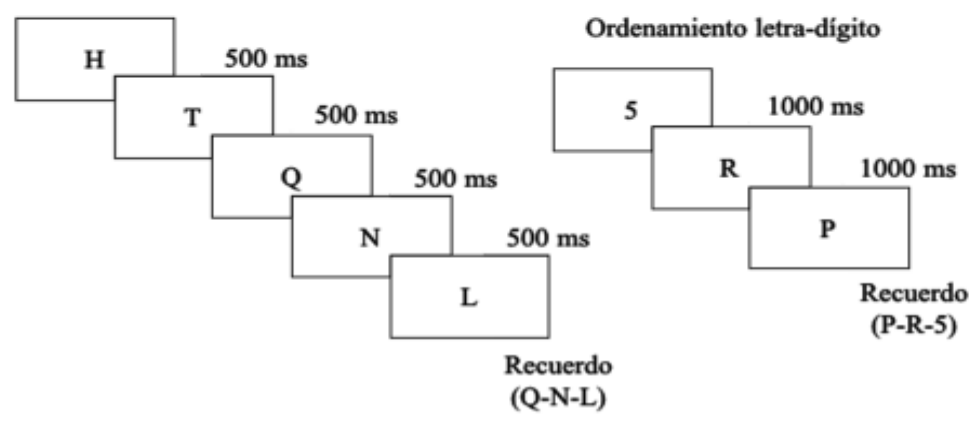

Figura 1. Ejemplo de Nivel 3 de las pruebas de memoria de trabajo verbal

\section{Tareas de atención sostenida}

Se administraron tareas para medir la capacidad de sostener la atención durante un periodo prolongado de tiempo: 1) Búsqueda de Símbolos (Wechsler, 2003), que consisten en presentar a un participante dos grupos de símbolos: un grupo objetivo y un grupo de búsqueda. La tarea consiste en indicar si alguno de los símbolos del grupo objetivo se encuentra en el grupo de búsqueda, en un tiempo determinado. 2) Claves de número (Wechsler, 2003), que consiste en presentar al participante una serie de números del uno al nueve, asociado cada uno a un símbolo específico. La tarea reside en completar en una matriz de 133 cuadrados en donde aparecen números ordenados aleatoriamente, los símbolos correspondientes a cada número durante un tiempo determinado.

\section{Cuestionario de inferencias explicativas- repositivas}

Para evaluar la generación de inferencias explicativas-repositivas se utilizó el texto Marte y la imaginación humana de Updlike (2009) y se confeccionó un cuestionario de preguntas sobre el contenido inferencial de dicho texto. Para armar el cuestionario, previamente se analizó en el texto las relaciones semánticas y conceptuales entre las ideas explícitas con el programa ETAT (Expository Text Analysis Tool; Vidal-Abarca et al., 2002). El análisis permitió identificar 11 oraciones que requerían, por parte del lector, explicaciones basadas en reposiciones de conceptos. Sobre la base de dichas oraciones se prepararon 11 preguntas explicativas de inferencias para ser administradas luego de la lectura 
del texto. El criterio de puntuación utilizado fue el siguiente: se asignó un punto a la respuesta que contenía referencia explícita a la inferencia, y cero puntos cuando el participante no contestaba la pregunta o la respondía de forma incorrecta. Por ejemplo ante la pregunta del texto de Marte: «¿Qué consecuencia tenía para los antiguos los movimientos del cuerpo celeste que denominaban Marte?», la respuesta correcta era que lo veían como violento o siniestro, diferente el del resto de las estrellas.

\section{Procedimiento}

Los participantes fueron testeados en dos sesiones. En la primera sesión, individual, completaron las tareas de memoria de trabajo y atención, donde se contrabalanceó el orden de presentación de las mismas. En la segunda sesión, grupal, los participantes leyeron el texto expositivo y completarán el cuestionario de inferencias.

\section{Análisis de datos}

En primer lugar, se realizaron las distribuciones de las pruebas administradas de memoria de trabajo, atención e inferencias. A continuación, se realizó un análisis de correlaciones entre las medidas de memoria de trabajo, inferencias y atención sostenida. En tercer lugar se pusieron a prueba un modelo para estudiar la relación entre la memoria de trabajo, la atención sostenida y la generación de inferencias empleando diferentes análisis de senderos (Arbuckle, 2014). Los índices de ajuste empleados para este análisis se basaron en convenciones y recomendaciones (Hair, Anderson, Tatham, \& Black, 1998; Jaccard \& Wan, 1996): Chi cuadrado $\left(\chi^{2}\right)$, Adjusted of Goodness of Fit Index (AGFI), Comparative Fit Index (CFI), Tucker-Lewis Index
(TLI) y Root Mean Squared Error of Approximation (RMSEA). El estadístico de Chi cuadrado evalúa la bondad de ajuste del modelo comparando la matriz de covarianza del modelo propuesto y la matriz de covarianza de los datos obtenidos. Un buen modelo muestra un estadístico no significativo (Hu \& Bentler, 1999). AGFI indica el grado de ajuste conjunto del modelo a los datos, un nivel aceptable y recomendado es un valor mayor o igual a .90 (Hair, et al., 1998). CFI, por otro lado, compara el ajuste del modelo existente con el de un modelo nulo, que asume que las variables latentes del modelo no están correlacionadas. CFI muestra un buen ajuste cuando su valor tiene es igual o mayor a .95 (Shumacker \& Lomax, 1996). TLI informa la proporción de la mejora en el ajuste del modelo propuesto en comparación con un modelo nulo, es una medida que no se ve afectada por tamaño de la muestra, valores superiores o iguales a .95 informan de un buen modelo (Shumacker \& Lomax, 1996). RMSEA indica el nivel de error entre el modelo y los datos, teniendo en cuenta los residuos. Cuando el valor de RMSEA es inferior a .06 indica un buen ajuste del modelo (Hu \& Bentler, 1998).

A partir de los puntajes obtenidos de las pruebas de memoria de trabajo, de atención sostenida e inferencias, se realizó en primer lugar, un análisis de sus distribuciones. De este análisis se pudo apreciar que, de todas las medidas administradas, solamente dos presentaron distribuciones que se alejaron significativamente de los percentiles teóricos de la distribución normal asintótica: amplitud rápida e inferencias explicativas-repositivas. En la tabla 1 se pueden observar los estadísticos descriptivos de todas las medidas analizadas. 
Tabla 1

Estadísticos descriptivos de las medidas analizadas

\begin{tabular}{lrrrrc}
\hline & $M$ & $D E$ & $A$ & $C$ & $K-S$ \\
\hline Amplitud de dígitos & 15.48 & 3.10 & -.24 & -.58 & 1.37 \\
Amplitud de letras & 13.93 & 2.82 & -.35 & -.51 & 1.11 \\
Ordenamiento letras-dígito & 10.34 & 3.31 & .09 & -.61 & 1.27 \\
Amplitud rápida & 5.62 & 2.06 & .24 & .38 & $1.47^{*}$ \\
Búsqueda de símbolos & 32.29 & 7.28 & .16 & .04 & .79 \\
Claves de números & 77.45 & 16.05 & .17 & 1.90 & .99 \\
Inferencias explicativas-repositivas & 3.21 & 1.87 & .85 & .51 & $1.85^{* *}$ \\
\hline
\end{tabular}

** $p<.01, * p<.05 ; M=$ media; $D E=$ desviación estándar; $A=$ asimetría; $C$ = curtósis.

Con en el objetivo de estudiar la relación entre las medidas de memoria de trabajo verbal, las medidas de atención sostenida y la generación de inferencias explicativas-repositivas en la comprensión de textos expositivos, se realizó el análisis de correlaciones utilizando el estadístico Rho de Spearman y el análisis de senderos (Path analysis). Para este último se llevó a cabo una normalización de las medidas de amplitud rápida e inferencias que mostraron distribuciones alejadas de la distribución normal, para ello se procedió a su transformación al logaritmo natural. En relación con las correlaciones, se puede observar que las medidas de memoria de trabajo presenta correlaciones positivas $y$ significativas de baja, media-baja y mediana intensidad con la generación de inferencias, y de las tareas de atención sostenida únicamente la prueba de búsqueda de símbolos se relacionó de manera positiva, significativa y con baja intensidad con la generación de inferencias (ver Tabla 2).

Tabla 2

Correlación entre las pruebas de memoria de trabajo, atención sostenida e inferencias

\begin{tabular}{lllllll}
\hline & 1. & 2. & 3. & 4. & 5. & 6. \\
\hline 1. Amplitud de dígitos & 1 & & & & \\
2. Amplitud de letras & $.44^{* *}$ & 1 & & & \\
3. Ordenamiento letras-dígito & $.49^{* *}$ & $.36^{* *}$ & 1 & & & \\
4. Amplitud rápida & $.54^{* *}$ & $.51^{* *}$ & $.43^{* *}$ & 1 & & \\
5. Búsqueda de símbolos & $.20^{*}$ & $.24^{* *}$ & $.40^{* *}$ & $.32^{* *}$ & 1 & \\
6. Claves de números & .07 & .03 & $.19^{*}$ & .15 & $.53^{* *}$ & 1 \\
7. Inferencias explicativas-repositivas & $.32^{* *}$ & $.19^{*}$ & $.21^{*}$ & $.47^{* *}$ & $.21^{*}$ & .09 \\
\hline$* * p<.01, * p<.05$ & & & & & &
\end{tabular}


Finalmente, se propuso un modelo donde la generación de inferencias explicativas-repositivas es explicada por el componente ejecutivo de la memoria de trabajo verbal (ejecutivo central), siendo el mismo afectado por el componente pasivo de almacenamiento de información verbal de la memoria de trabajo (bucle fonológico), y por la atención sostenida. Esta relación es propuesta debido a que las tareas de almacenamiento y procesamiento concurrente de información son saturadas por procesos de almacenamiento de información verbal y por recursos atencionales. En la figura 2 que se presenta a continuación se muestra el modelo testeado.

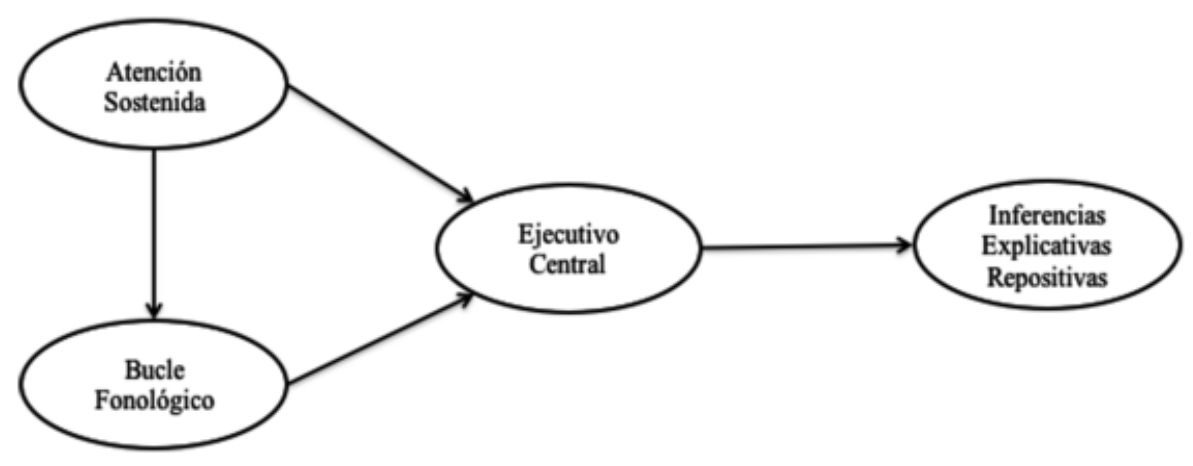

Figura 2. Modelos relación entre los componentes de la memoria de trabajo, la atención sostenida y la generación de inferencias.

El análisis de ecuaciones estructurales se realizó a partir de la estimación de máxima verosimilitud entre los ítems como input para el análisis (Harrington, 2008). Los resultados obtenidos del análisis de mostraron que el modelo propuesto presentó un muy buen ajuste a los datos $\chi_{(11)}^{2}=13.76, p=.25, A G F I$ $=.92, T L I=.97, C F I=.99$ y RMSEA (Root Mean Square Error of Aproximation) $=.05$. Al mirar los pesos de regresión del modelo, se observa que el componente ejecutivo de la memoria de trabajo verbal (ejecutivo central) tiene un efecto directo de mediana intensidad sobre la generación de inferencias explicativas-repositivas $(\beta=.44, p<.01)$, el componente pasivo de almacenamiento de información verbal de la memoria de trabajo (bucle fonológico) tiene un efecto directo de alta intensidad sobre el componente ejecutivo de la memoria de trabajo $(\beta=.89, p<.01)$. La atención sostenida tiene un efecto directo de baja intensidad y significativo sobre el componente pasivo de almacenamiento de información de la memoria de trabajo $(\beta=.30, p<$ .01), como también sobre el componente ejecutivo de la misma $(\beta=.20, p<.05)$. Al analizar los efectos indirectos de la atención sostenida y del almacenamiento de información verbal de la memoria de trabajo sobre la generación de inferencias, se observa que el componente ejecutivo de la misma, tiene un rol mediador en la relación entre estas últimas y la generación de inferencias, ya que el componente pasivo de almacenamiento tiene un efecto indirecto de mediana intensidad $(\beta=.39, p<$ .01) sobre la generación de inferencias, y la atención sostenida tiene un efecto indirecto de baja intensidad $(\beta=.20, p<.05)$ (ver Figura 3). 


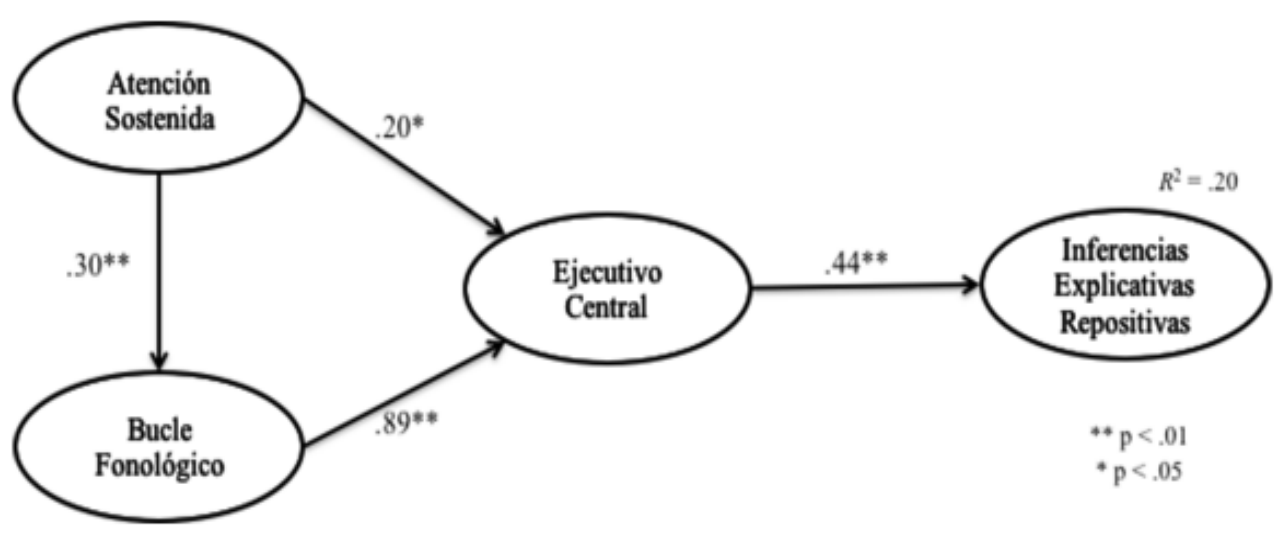

Figura 3. Modelo de la relación entre la memoria de trabajo y la atención sostenida sobre la generación de inferencias.

\section{Discusión}

El objetivo principal de este trabajo consistió en estudiar el rol de la memoria de trabajo, específicamente los subsistemas de almacenamiento de información verbal (bucle fonológico) y el sistema implicado en almacenamiento y procesamiento concurrente de información verbal (ejecutivo central), la capacidad de sostener la atención sobre la generación de inferencias explicativas-repositivas en la comprensión de textos expositivos. Con este fin, se administraron a 120 alumnos universitarios cuatro pruebas de memoria de trabajo de la Batería Informatizada de Memoria de Trabajo Verbal (Barreyro et al., s.f.) -dos pruebas de almacenamiento de información verbal y dos pruebas de almacenamiento y procesamiento concurrente-, junto con dos pruebas de atención sostenida, un texto expositivo y un cuestionario de inferencias explicativas-repositivas.

El resultado del análisis de correlaciones muestra que el cuestionario de inferencias está asociado positiva y significativamente a las pruebas de memoria de trabajo, pero únicamente a una de las pruebas de atención (búsqueda de símbolos). Esto indica que la memoria de trabajo es un sistema implicado en la generación de inferencias explicativas-repositivas. Esto es, la reposición de conceptos necesarios para dotar de mayor coherencia a la oración que se está leyendo, durante la comprensión de un texto esta soportada por recursos de almacenamiento y de almacenamiento y procesamiento concurrente de memoria de trabajo. Resultados similares han sido encontrados en relación a la comprensión de textos en general tanto en niños como en adultos (Hannon, 2012; Loosli, Buschkuehl, Perrig, \& Jaeggi, 2012; McVay \& Kane, 2012; Wang \& Gathercole, 2013) y también vinculados a la generación de inferencias (Barreyro et al., 2012; Currie \& Cain, 2015; Freed \& Cain, 2016; Pérez, Paolieri, Macizo, \& Bajo, 2014; Prat et al., 2016).

El análisis de ecuaciones estructurales (análisis de senderos) muestra en principio un muy buen ajuste del modelo propuesto a los datos obtenidos. El modelo propuesto plantea que la generación de inferencias es explicada por el componente ejecutivo de la memoria de trabajo, y este por el componente de almacenamiento de información verbal (bucle fonológico) y por la atención sostenida. La propuesta de este modelo es coherente con las investigaciones que indican el rol de la memoria de trabajo sobre la comprensión de textos y la generación de inferencias, empleando modelos de ecuaciones estructurales (McVay \& Kane, 2012), y también es coherente en relación a la relación entre la memoria de trabajo y 
la capacidad de sostener la atención (Baddeley, 2010, 2012; Cowan, 2012).

En relación a los resultados obtenidos del análisis de ecuaciones estructurales se puede apreciar que el componente ejecutivo de la memoria de trabajo (ejecutivo central) tiene un efecto directo de mediana intensidad sobre la generación de inferencias, mientras el almacenamiento de información verbal tienen una incidencia indirecta de mediana intensidad y la atención sostenida tienen una incidencia indirecta de baja intensidad, en ambos casos mediado por el componente ejecutivo, y en el caso de la atención mediado también por el componente pasivo de la memoria de trabajo.

Los resultados son coherentes en relación a los componentes de memoria involucrados, tanto en la comprensión de textos, como en relación a la generación de inferencias (Calvo, 2004; Carretti, Borella, Cornoldi, \& De Beni, 2009; Prat et al., 2016; van den Broek, Mouw, \& Kraal, 2016), y permiten reforzar la afirmación que sostiene que las diferencias individuales en la generación de inferencias están vinculadas a las diferencias inviduales en la memoria de trabajo, tanto en la capacidad de almacenamiento de información como en la capacidad de de almacenamiento y procesamiento concurrente.

En relación con la capacidad de sostener la atención, los resultados muestran una incidencia indirecta sobre la generación de inferencias, lo que permitiría afirmar que la capacidad para reponer un concepto previamente presentado en el texto, para establecer vinculos entre la información anteriormente presentada y la información que se está leyendo, está siendo soportada indirectamente por los recursos atencionales que permiten mantener durante un periodo prolongado de tiempo el foco de atención sobre una tarea, mediado por la memoria de trabajo.

Los resultados del presente estudio permiten comprender que si bien la memoria de trabajo y la atención sostenida tienen una relación con la generación de inferencias y específicamente en la explicación de una oración mediante la reposición de conceptos leídos previamente en el texto, hay una relación de interacción entre la memoria de trabajo y la atención sostenida que se relaciona con la habilidad para generar inferencias, que puede indicar que los recursos atencionales para sostener el foco de la atención en la lectura, como lo marcan varias investigaciones previas en relación a la dispersión (McVay \& Kane, 2012; Unsworth \& McMillan, 2013) tienen una implicancia importante para la tarea, pero dependen de la capacidad de la memoria de trabajo para llevar a cabo la tarea de activación y recuperación de información desde la memoria de largo plazo del texto con el objetivo de comprender lo que se está leyendo (Kane \& Engle, 2000; Kintsch, Patel, \& Ericsson, 1999). Este fenómeno de interacción que es observado en el modelo propuesto debería ser ampliado para llegar a un resultado más fiable que el obtenido en este trabajo.

Las limitaciones de este estudio están vinculadas en principio a las características de la muestra, ya que una muestra mayor, con una mejor distribución entre participantes (respecto al género) podría permitir aseverar con mayor firmeza los resultados obtenidos. Esto se podría observar también si se plantea un estudio en el que se emplee una gama mayor de textos expositivos y se evalúen más componentes atencionales.

Futuras investigaciones a partir del presente trabajo podrían replicar este resultado o el modelo presentado en la aplicación de otros tipos de textos, como los narrativos y descriptivos, y otros tipos de textos expositivos, como así también estudiar el fenómeno en otro tipo de inferencias. A su vez, nuevas investigaciones podrían indagar características del lector, como diferencias en la edad de los lectores (participantes con edades tempranas, adultos y en la vejez), la habilidad lectora y las estrategias empleadas por los lectores. 


\section{Referencias}

Abusamra, V., \& Joanette, Y. (2012). Lectura, escritura y comprensión de textos: aspectos cognitivos de una habilidad cultural. Neuropsicologia Latinoamericana, 4(1), $1-4$.

Arbuckle, J. L. (2014). IBM SPSS AMOS 22 User's Guide. Mount Pleasant, SC: Amos Development Corporation.

Awh, E., \& Jonides, J. (2001). Overlapping mechanisms of attention and spatial working memory. Trends in Cognitive Sciences, 5(3), 119-126.

Baddeley, A. D. (2010). Working memory. Current Biology, 20(4), 136-140. doi: 10.1016/j.cub.2009.12.014

Baddeley, A. D. (2012). Working Memory: Theories, Models, and Controversies. Annual Review of Psychology, 63, 1-29. doi: 10.1146/annurev-psych-1207 10-100422

Baddeley, A. D., \& Hitch, G. J. (1974). Working memory. In G. H. Bower (Ed.), The Psychology of Learning and Motivation: Advances in Research and Theory (Vol. 8, pp. 47-90). New York: Academic Press.

Barreyro, J. P., Cevasco, J., Burin, D. I., \& Molinari, C. (2012). Working memory capacity and individual differences in the making of reinstatement and elaborative inferences. Spanish Journal of Psychology, 15(2), 471-479. doi: 10.5209/rev_SJOP.2012.v15.n2.38857

Barreyro, J. P., Injoque Ricle, I., Formoso, J., \& Burin, D. (s.f.). Computerized Working Memory Battery (BIMeTV): Studying the Relation between Working Memory, Verbal Reasoning and Reading Comprehension. Manuscrito presentado para su publicación.

Barth, A., Barnes, M. A., Francis, D., York, M., \& Vaughn, S. (2015). Bridging inferences among adequate and struggling adolescent comprehenders and relations to reading comprehension. Reading and Writing, 28(5), 587-609. doi: 10.1007/s11145-014-9540-1

Bohn-Gettler, C. M., \& Kendeou, P. (2014). The interplay of reader goals, working memory, and text structure during reading. Contemporary Educational Psychology, 39(3), 206-219. doi: 10.1016/j.cedpsych.20 14.05.003
Brock, S. E., \& Knapp, P. K. (1996). Reading comprehension abilities of children with attentiondeficit/hyperactivity disorder. Journal of Attention Disorders, 1(13), 173-185.

Budd, D., Whitney, P., \& Turley, K. (1995). Individual differences in working memory strategies for reading expository text. Memory \& Cognition, 23, 735-748. doi: 10.3758/BF03200926

Calvo, M. G. (2004). Relative contribution of vocabulary knowledge and working memory span to elaborative inferences in reading. Learning and Individual Differences, 15, 53-65. doi: 10.1016/j.lindif.2004.07.002

Carlson, S. E., van den Broek, P., McMaster, K., Rapp, D. N., Bohn-Gettler, C. M., Kendeou, P., \& White, M. J. (2014). Effects of comprehension skill on inference generation during reading. International Journal of Disability, Development and Education, 61(3), 258274. doi: 10.1080/1034912X.2014.934004

Carretti, B., Borella, E., Cornoldi, C., \& De Beni, R. (2009). Role of working memory in explaining the performance of individuals with specific reading comprehension difficulties: A meta-analysis. Learning and Individual Differences, 19, 246-251. doi: 10.1016/j.lindif.200810.002

Cornoldi, C., \& Oakhill, J. V. (2013). Reading comprehension difficulties: Processes and intervention. Oxford, UK: Routledge.

Cowan, N. (2012). Working memory capacity. New York: Psychology press.

Currie, N. K., \& Cain, K. (2015). Children's inference generation: The role of vocabulary and working memory. Journal of Experimental Child Psychology, 137, 57-75. doi: 10.1016/j.jecp.2015.03.005

Diamond, A., \& Goldman-Rakic, P. S. (1989). Comparison of human infants and rhesus monkeys on Piaget's tasks: Evidence of dependence on dorsolateral prefrontal cortex. Experimental Brain Research, 74(9), 24-40.

Elbro, C., \& Buch-Iversen, I. (2013). Activation of background knowledge for inference making: effects on reading comprehension. Scientific Studies of Reading, 17(6), 435-452. doi: 10.1080/10888438.2013. 774005 
Freed, J., \& Cain, K. (2016). Assessing school-aged children's inference-making: the effect of story test format in listening comprehension. International Journal of Language \& Comunication Disorders, 52, 95-105. doi: 10.1111/1460-6984.12260

Graesser, A. C., \& Bertus, E. L. (1998). The construction of causal inferences while reading expository texts on science and technology. Scientific Studies of Reading, 2(3), 247-269. doi: 10.1207/s1532799xssr0203_4

Graesser, A. C., Leon, J. A., \& Otero, J. C. (2002). Introduction to the psychology of science text comprehension. In J. Otero, J. A. Leon \& A. C. Graesser (Eds.), The psychology of science text comprehension (pp. 1-15). Mahwah, NJ: Erlbaum.

Graesser, A. C., Singer, M., \& Trabasso, T. (1994). Constructing inferences during narrative text comprehension. Psychological Review, 101, 371-395.

Hair, F., Anderson, R. E., Tatham, R. L., \& Black, W. C. (1998). Multivariate data analysis with readings. New Jersey, NJ: Prentice Hall.

Hannon, B. (2012). Understanding the Relative Contributions of Lower Level Word Processes, Higher Level Processes, and Working Memory to Reading Comprehension Performance in Proficient Adult Readers. Reading Research Quarterly, 47(2), 125-152. doi: 10.1598/0710.33

Harrington, D. (2008). Confirmatory factor analysis. Oxford: Oxford University Press.

Hecker, L., Burns, L., Katz, L., Elkind, J., \& Elkind, K. (2002). Benefits of assistive reading software for students with attention disorders. Annals of Dyslexia, 52(1), 243-272.

Hu, L., \& Bentler, P. M. (1998). Fit indices in covariance structure modeling: Sensitivity to underparameterized model misspecification. Psychological Methods, 3, 424-453. doi: 10.1037/1082-989X.3.4.424

Hu, L., \& Bentler, P. M. (1999). Cut-off criteria for fit indexes in covariance structure analysis: Conventional criteria versus new alternatives. Structural Equation Modeling, 6, 1-55. doi: 10.1080/10705519909540118

Jaccard, J., \& Wan, C. K. (1996). LISREL approaches to interaction effects in multiple regression. Thousand Oaks, CA: Sage Publications.
Kane, M. J., \& Engle, R. W. (2000). Working-memory capacity, proactive interference, and divided attention: limits on long-term memory retrieval. Journal of Experimental Psychology: Learning, Memory, and Cognition, 26(2), 336-358. doi: 10.1037/0278-7393. 26.2.336

Kendeou, P., Smith, E. R., \& O’Brien, E. J. (2013). Updating during reading comprehension: Why causality matters. Journal of Experimental Psychology. Learning, Memory, and Cognition, 39, 854-865. doi: 10.1037/a00 29468

Kintsch, W., Patel, V. L., \& Ericsson, K. A. (1999). The role of long-term working memory in text comprehension. Psychologia, 42(4), 186-198.

Kintsch, W., \& van Dijk, T. A. (1978). Towards a model of text comprehension and production. Psychological Review, 85, 363-394. doi: 10.1037/0033-295X.85.5.363

Leon, J. A., \& Peñalba, G. E. (2002). Understanding Causality and Temporal Sequence in Scientific Discourse. In J. Otero, J. A. Leon \& A. Graesser (Eds.), The Psychology of Science Text Comprehension (pp. 155-178). Mahwah, NJ: Lawrence Erlabaum.

Loosli, S. V., Buschkuehl, M., Perrig, W. J., \& Jaeggi, S. M. (2012). Working memory training improves reading processes in typically developing children. Child Neuropsychology, 18(1), 62-78. doi: 10.1080/09297049. 2011.575772

McVay, J. C., \& Kane, M. J. (2012). Why does working memory capacity predict variation in reading comprehension? On the influence of mind wandering and executive attention. Journal of Experimental Psychology: General, 141(2), 302-320. doi: 10.1037/a00 25250

Pérez, A. I., Paolieri, D., Macizo, P., \& Bajo, T. (2014). The role of working memory in inferential sentence comprehension. Cognitive processing, 15(3), 405-413. doi: 10.1007/s10339-014-0611-7

Prat, C. S., Seo, R., \& , \& Yamasaki, B. L. (2016). The Role of Individual Differences in Working Memory Capacity on Reading Comprehension Ability. In P. Afflerbach (Ed.), Handbook of Individual Differences in Reading: Reader, Text, and Context (pp. 331-347). New York: Routledge. 
Rebollo, M. A., \& Montiel, S. (2006). Atención y Funciones Ejecutivas. Revista de Neuropsicología, 42(3), 3-7.

Saux, G., Irrazabal, N. \& Burin, D.I. (2014). Comprensión de textos de ciencias en estudiantes universitarios: generación de inferencias causales durante la lectura. Liberabit, 20(2), 305-313.

Shumacker, R. E., \& Lomax, R. G. (1996). A beginner guide to structural equation modeling: Mahwah, NJ: Erlbaum.

Singer, M., Harkness, D., \& Stewart, T. (1997). Constructing inferences in expository text comprehension. Discourse Processes, 24, 199-228.

Singer, M., \& O’Connell, G. (2003). Robust inference processes in expository text comprehension. European Journal of Cognitive Psychology, 15(4), 607-631. doi: 10.1080/095414400340000079

Snow, C. (2002). Reading for understanding: Toward an $R \& D$ program in reading comprehension. Arlington, VA.

Unsworth, N., \& McMillan, B. D. (2013). Mind wandering and reading comprehension: Examining the roles of working memory capacity, interest, motivation, and topic experience. Journal of Experimental Psychology: Learning, Memory, and Cognition, 39(3), 832-842. doi: 10.1037/a0029669

Updlike, J. (2009). Marte, regreso al planeta rojo. National Geographic España, 24(1), 2-19.

van den Broek, P., \& Kendeou, P. (2008). Cognitive processes in comprehension of science texts: The role of co-activation in confronting misconceptions. Applied Cognitive Psychology, 22, 335-351. doi: 10.100 2/acp.1418

van den Broek, P., Mouw, J. M., \& Kraal, A. (2016). Individual Differences in Reading Comprehension. In P. Afflerbach (Ed.), Handbook of Individual Differences in Reading: Reader, Text, and Context (pp. 138-150). New York, NY: Routledge.

van den Broek, P., Risden, K. C., Fletcher, C. R., \& Thurlow, R. (1996). A «landscape» view of reading: Fluctuating patterns of activation and the construction of a stable memory representation. . In B. K. Britton \& A. C. Graesser (Eds.), Models of understanding text (pp. 165-187). Mahwah, NJ: Lawrence Erlbaum.

van den Broek, P., Rohleder, L., \& Narváez, D. (1996). Causal inferences in the comprehension of literary text. In R. J. Kreuz \& M. S. MacNealy (Eds.), Empirical approaches to literature and aesthetics. Norwood, NJ: Ablex Publishing Corporation.

van den Broek, P., Virtue, S., Everson, M., Tzeng, Y., \& Sung, Y. C. (2002). Comprehension and memory of science texts: Inferential processes and the construction of a mental representation. In J. Otero, J. A. Leon \& A. C. Graesser (Eds.), The Psychology of Science Text Comprehension (pp. 131-154). Mahwah, NJ: Lawrence Erlbaum Associates, Inc.

Van Dijk, T., \& Kintsch, W. (1983). Strategies of discourse comprehension. New York, NY: Academic Press.

Van Dyke, J. A., Johns, C. L., \& Kukona, A. (2014). Low working memory capacity is only spuriously related to poor reading comprehension. Cognition, 131(3), 373403. doi: 10.1016/j.cognition.2014.01.007

Vidal-Abarca, E., Martínez, E., \& Gilabert, R. (2000). Two procedures to improve instructional text: Effects on memory and learning. Journal of Educational Psychology, 92, 107-116. doi: 10.1037/0022-0663. 92.1.107

Vidal-Abarca, E., Reyes, H., Gilabert, R., Calpe, J., Soria, E., \& Graesser, A. C. (2002). ETAT: Expository Text Analysis Tool. Behavior Research Methods, Instruments, \& Computers, 34, 93-107. doi: 10.3758/BF 03195428

Wang, S., \& Gathercole, S. E. (2013). Working memory deficits in children with reading difficulties: memory span and dual task coordination. Journal of Experimental Child Csychology, 115(1), 188-197. doi: 10.1016/j.jecp.20 12.11.015

Wechsler, D. (2003). WAIS III: Test de Inteligencia para Adultos. Buenos Aires, AR: Paidós. 


\section{Juan Pablo Barreyro}

Departamento de Procesos Básicos, Instituto de Investigaciones, Facultad de Psicología, Universidad de Buenos Aires - CONICET Doctor en Psicología de la Universidad de Buenos Aires, Investigador Adjunto de la carrera del investigador científico de la comisión nacional de investigación científica y tecnológica (CONICET).

Autor corresponsal: jbarreyro@psi.uba.ar

Irene Injoque-Ricle

Departamento de Procesos Básicos, Instituto de Investigaciones, Facultad de Psicología, Universidad de Buenos Aires - CONICET

Doctora en Salud Mental de la Universidad de Buenos Aires, Investigadora Adjunta de la carrera del investigador científico de la comisión nacional de investigación científica y tecnológica (CONICET).

iinjoque@psi.uba.ar

\section{Jésica Formoso}

Departamento de Procesos Básicos, Instituto de Investigaciones, Facultad de Psicología, Universidad de Buenos Aires - CONICET Doctora en Salud Mental de la Universidad de Buenos Aires, Becaria Post-doctoral interna de la comisión nacional de investigación científica y tecnológica (CONICET).

jformoso@psi.uba.ar

\section{Debora I. Burin}

Departamento de Procesos Básicos, Instituto de Investigaciones, Facultad de Psicología, Universidad de Buenos Aires - CONICET Doctora en Psicología de la Universidad de Salamanca, Investigadora Independiente de la carrera del investigador científico de la comisión nacional de investigación científica y tecnológica (CONICET). 